\title{
A Review on Nutrition and Epigenetics with their Possible Influence on Cancer Development
}

\author{
Sharmin Sultana and Rashed Noor* \\ Department of Life Sciences (DLS), School of Environment and Life Sciences, (SELS), Independent University, Bangladesh \\ *Corresponding author: Rashed Noor, Department of Life Sciences (DLS), School of Environment and Life Sciences, \\ (SELS), Independent University, Bangladesh
}

\section{ARTICLE INFO}

Received: 幽 February 01, 2021

Published: 㓞 February 09, 2021

Citation: Sharmin Sultana, Rashed Noor A Review on Nutrition and Epigenetics with their Possible Influence on Cancer Development. Biomed J Sci \& Tech Res 33(5)-2021. BJSTR. MS.ID.005454.

Keywords: Nutrition; Diet; Epigenetic Modifications; Cancer

\section{ABSTRACT}

Bioactive substances present in dietary components can modify the way of gene expression. This can take place through epigenetic changes which may be reversible. Epigenetic alterations which may have arisen due to the intake of varieties of nutritional components, have been focused on the current review to infer their possible effects on the onset of physiological dysbiosis such as cancer.

Abbreviations: SFN: Sulforaphane; EGCG: Epigallocatechin-3-Gallate; hTERT: Human Telomerase Reverse Transcriptase; RNAi: RNA Interference; DNMT: DNA Methyltransferases; HATs: Histone Acetyltransferases; HDACs: Histone Deactylasesl; miRNA: Micro RNA; siRNA: Small Interfering RNA; CR: Caloric Restriction; SAM: S-Adenosylmethionine

\section{Mini Review}

Epigenetics is an exciting area of study in today's biological field. Epigenetics is commonly known as the study where modifications are brought about in gene expression by altering the way chromatin is arranged without causing any change in the existing DNA sequences; i.e., the non-Mendelian inheritance of DNA modifications [1-3]. Epigenetic changes can be caused by factors such as environmental factors, dietary intake, medications etc., and very often, these changes can be reversible [3]. Epigenetic changes during early pregnancy are quite unique; however, the epigenetic changes that take place during the livelihood of a person are often contributed especially by the dietary intakes of an individual [3]. These modifications aspects associated with epigenetics may eventually lead to the onset of fatal diseases even cancer [3]. An array of phytochemicals like the sulforaphane (SFN) from cruciferous vegetables (like broccoli, cauliflower, cabbage, etc.) and especially green tea having epigallocatechin-3-gallate (EGCG) have been shown to possess both the anti-oxidant and the anticancer epigenetic effects thereby suppressing the telomerase, encoded by the human telomerase reverse transcriptase (hTERT) which is central to tumor progression; and hence they may be effective for the prevention of the epigenetic abnormalities of other age-related complications including cancer [2].
Calories from the dietary intake may also be beneficial in counteracting cancer as well as ranging the average lifespan [2]. This is interesting to ponder that only the genotype is always not responsible for the onset of cancer; rather, the dietary intake may largely influence in this regard [3]. Previous studies showed the interrelationship between the genomic organization, epigenetics, the dietary nutrition instigating breast cancer, colon cancers, and colorectal cancers $[2,3]$. In the current review, further incremental inputs on the existing knowledge have been given focusing on the connection between epigenetics and dietary intake triggering the commencement of cancer. Some of the aspects of dietary intake on epigenetic modification and cancer development have been highlighted.

\section{Epigenetic Modifications and Cancer}

The study of transcriptional regulation together with the dysregulation in cancer epigenetic mechanisms has largely drawn the interests of scientists [2-6]. An interesting aspect is to be noted that involves the epigenetic drug therapy which may hinder the cancer progression probably by repressing the associated oncogenes with the concomitant activation of the tumor-suppressors [5]. Several mechanisms during the epigenetic 
transcriptional control including the DNA methylation, covalent histone modification/ exchange which is afterward read by the protein recognition modules, the involvement of the noncoding RNAs, and the amendment method employed by the ATP-dependent chromatin remodelers have been so far reported [3-5]. Some of the most commonly known mechanisms; i.e., DNA methylation, histone modification, and RNA interference (RNAi) by the small non-coding RNAs are discussed below $[3,4]$.

\section{DNA Methylation Leading to the Development of Cancer}

In DNA methylation, a methyl group is added by means of the DNA methyltransferases (DNMT) to the carbon-5 of the cytosine's pyrimidine ring, and these cytosines are usually ones which are present in $\mathrm{CpG}$ islands [2,3]. Methylation of gene regulatory regions are likely to cause less transcription from the specific promoter [2]. Cancer can occur due to both hypomethylation and hypermethylation of these cytosine residues of the $\mathrm{CpG}$ islands $[3,4]$. The hypomethylation of the $\mathrm{CpG}$ sites present in intergenic regions result in the chromatin thread to become loosely packed, and this can lead to the transcription of the DNA [3]. Moreover, hypomethylation can directly trigger the oncogenes to get activated, resulting in tumor formation [4]. On the other hand, hypermethylation of promoter regions of tumor suppressor genes can lead to less transcription to ensue from the promoter, and this too may result in cancer to be developed $[2,4]$.

Histone Modifications Leading to the Development of Cancer

The next epigenetic modification to be discussed is the histone modification involving histone methylation, histone acetylation, phosphorylation, and ubiquitination [2]. Histones are indeed proteins around which the DNA wraps itself to stay in its condensed chromosome form [4]. When histone modification arises, the histone tails containing different amino acids get modified [4]. Enzymes facilitating the histone modifications have been shown to be related to those causing DNA methylation of which histone acetyltransferases (HATs) and the histone deacetylases (HDACs) are important because of their involvement in triggering cancer and aging [2]. During histone acetylation, acetyl group can be added to various regions of the histone tails, especially that where lysine residues are present [5]. It is known that lysine is a positively charged amino acid and DNA is negatively charged, and they are attracted to each other via electrostatic attraction [5] Therefore, when lysine residues get acetylated, it results in those lysine groups of the histone protein to no longer be attracted to the parent, supercoiled DNA, and hence instigating that DNA to undergo a relaxed conformation, and thus, in such a conformation transcription mode, the gene expression can occur at a higher frequency which can eventually lead to cancer $[3,5]$.

\section{Small RNAs and the Development of Cancer}

Yet another epigenetic modification is linked to small, singlestranded non-coding RNAs (21-23 nucleotides in length); i.e., the
microRNA (miRNA), small interfering RNA (siRNA) etc. which may suppress the mRNA expression [2]. It has also been found that the small non-coding RNAs, especially the miRNAs' lack of regulation of transcriptional activities can ultimately lead to cancer development [3]. An example of such a case was observed in cervical and gastric cancers [3]

\section{Influence of Nutrition on Epigenetic Modification and Cancer}

Nutritional food intake may help in preventing cancer in many instances; however, sometimes the way the food is processed for intake can lead to the onset of cancer. More specifically, a delay in the aging consequence, as well as the hindrance of age-related diseases (possibly by regulating the distribution of 5-methylcytosine and thereby maintaining the decrease in global DNA methylation patterns) can be facilitated by epigenetic modifications instigated by nutrition in the form of caloric restriction (CR) by $30-40 \%$ which ultimately imparts a sustainability in chromatin function [4]. Besides, a range of dietary nutrients may constitute the bioactive compounds which are effective in preventing cancer as well as these may also facilitate satisfactory epigenetic changes [2]. For instance, folate is a well-known and well-studied component, with the help of which one-carbon metabolism can take place [6]. Through this method, S-adenosylmethionine (SAM) gets formed and this is a universal methyl donor. The addition of these methyl groups to the DNA can lead to DNA methylation [6]. Besides folate, some other nutrients involved in this mechanism are methionine and few different variants of vitamin B [6]. These are known to be some of the most important cofactors involved in the one-carbon metabolism mechanism [6].

This is also to be noted that HDACs are usually elevated in the cancer cells; and their inhibition may be facilitated by the SFN from the intake of diet consisting of the cruciferous vegetables [2]. However, the accurate effect of folate on epigenetics and cancer needs further extended studies and evaluation. There are other methyl group donors besides SAM, such as choline and betaine, which can further result in DNA methylation [6]. It has been found that dietary intake of folate can result in a decreased risk of having colorectal and hematopoietic cancer [6]. In contrast, deficiency of folate can lead to global DNA hypomethylation, but if folate is administered in sufficient quantity to those subjects, it can reverse the negative effects of folate deficiency by the restoration of the original DNA methylation status [6]. One experimental example can be drawn in this perspective. A group of rats was given diet deficient in methyl and they portrayed hypomethylation in the entire genome and also in oncogenic promoters (c-myc, c-fos and H-Ras) in the liver [7]. This issue was reverted back when the rats were given a diet adequate with methyl components [8]. Another study showed that low folate and high alcohol diet resulted in tumor suppressor genes- $p 14 A R F, P 16 I N K 4 A, h M L H 1, M G M T$, and $R A S S F 1 A$, to get more hypermethylated in their promoter regions for patients with colorectal cancer $[9,10]$. 


\section{Nutrients Affecting Histone Modification}

As mentioned above, DNA condenses down by wrapping itself around histone proteins. By using covalent modifications such as methylation, acetylation etc., amino acids sequences present on its histone tails can be altered [4]. These modifications determine how tightly the DNA is wrapped around the histone proteins, which eventually regulate gene expression. Since these modifications are reversible in nature, specific nutrients or substances can be used to target the unusual histone modifications and reverse those to prevent cancer from ensuing from those sites [6]. A study showed that cancer can arise due to the deacetylation of the histone $\mathrm{H} 4$ protein [6]. Such an event is facilitated by the enzyme histone deacetylase (HDAC) which further results in the chromatin to become inactive as the proteins involved in transcription can no longer access the promoter region of the gene [11]. A diet containing the epigenetic-modifying nutrients may largely influence the prevalence of cancer [2]. As stated earlier, substances such as SFN (especially from broccoli and its sprouts) have been found to inhibit HDACs by forming a weak ligand interaction with them, and this phenomenon was studied for various kinds of cancer such as colorectal cancer, breast cancer and prostate cancer [6].

Another interesting component curcumin, the active component of turmeric, has also been found to aid the acetylation of $\mathrm{H} 3$ and H4 histone proteins, and contribute to the apoptosis of prostate cancer cells by involving $B c l-2$ gene family and $p 53[6,10,12,13]$. Yet, another component, genistein found in fruits and vegetables, has proven to decrease the acetylation of the $\mathrm{H} 3$ histone proteins by inhibiting the HDACs [12]. Butyrate is another fascinating compound, present in butter, is also involved in the inhibition of HDACs [12]. As mentioned above, the EGCG of green tea may impart a range of impact on epigenetic mechanisms such as inhibition of DNMT, HDAC, and HAT as well as the expression of the non-coding RNAs [2,4]. Due to these mechanisms, the consumption of EGCG has shown promising results in inhibiting a number of different cancers such as oral, breast, prostate, colorectal cancers and more [4]. To be more specific, EGCG is proven to downregulate the expression of hTERT in MCF-7 human breast cancer cells due to demethylation of TERT promoter and acetylation of H3 Lys9 acetylation [4].

\section{Challenges of and Future Perspectives}

There are several challenges and gaps which have to be addressed in order to possibly use various of the above-mentioned bioactive molecules to deal with different cancer types. One such issue is the optimization of the effective dose in order to prevent cancer [6]. Another important aspect is to consider that the dietary substances may affect the entire epigenome in a very non-selective manner, thereby causing the other uncalled-for diseases [6]. Furthermore, to figure out the actual efficacy of such an intervention of the effectiveness of the epigenetic drugs, a suitable sample size would be required along with a plethora of resources, which would indefinitely become an extremely expensive process making it a rather difficult area to finance, study on and henceforth, investigate
[6]. Despite the challenges of the investigations, an important aspect of related future studies should be regarding methods for the genome-wide analysis and high-throughput assays to understand more about the interaction between the bioactive dietary active components with various types of epigenetic modifications [12].

\section{Conclusion}

The ability of dietary components to bring about the epigenetic changes in cancer cells is an important area of study to understand mechanisms of preventing cancer. The current review unassumingly highlighted on the aspects of nutrition's influence on epigenetic modification leading to the development of cancer-based on the previously published literature. The information given here may open doors to the nutritionists and dieticians to think about the genetic aspects of the prevention of aging and cancer.

\section{Conflict of Interest}

None.

\section{Funding}

None.

\section{References}

1. Tiffon C (2018) The Impact of Nutrition and Environmental Epigenetics on Human Health and Disease. Int J Mol Sci 19(11): 3425.

2. Tollefsbol TO (2014) Dietary epigenetics in cancer and aging. Cancer Treat Res 159: 257-267.

3. Bishop KS, Ferguson LR (2015) The interaction between epigenetics, nutrition and the development of cancer. Nutrients 7(2): 922-947.

4. Daniel M, Tollefsbol TO (2015) Epigenetic linkage of aging, cancer and nutrition. J Exp Biol 218(Pt 1): 59-70.

5. Audia JE, Campbell RM (2016) Histone Modifications and Cancer. Cold Spring Harb Perspect Biol 8(4): a019521.

6. Chen J, Xu X (2010) Diet, epigenetic, and cancer prevention. Adv Genet 71: 237-55.

7. Christman JK, Sheikhnejad G, Dizik M, Abileah S, Wainfan E (1993) Reversibility of changes in nucleic acid methylation and gene expression induced in rat liver by severe dietary methyl deficiency. Carcinogenesis 14(4): 551-557.

8. Pogribny IP, Shpyleva SI, Muskhelishvili L, Bagnyukova TV, James SJ, et al. (2009) Role of DNA damage and alterations in cytosine DNA methylation in rat liver carcinogenesis induced by a methyl-deficient diet. Mutat Res 669(1-2): 56-62.

9. Shin W, Yan J, Abratte CM, Vermeylen F, Caudill MA (2010) Choline intake exceeding current dietary recommendations preserves markers of cellular methylation in a genetic subgroup of folate-compromised men. J Nutr 140(5): 975-980.

10. Zakaria A, Asaduzzaman SAI, Nahar Z, Snigdha HJ, Murshed T, et al. (2021) A short review of the genes involved in the development and progression of colorectal cancer. BIOCELL, p. 1-5.

11. Parbin S, Kar S, Shilpi A, Sengupta D, Deb M, et al. (2014) Histone deacetylases: a saga of perturbed acetylation homeostasis in cancer. J Histochem Cytochem 62(1): 11-33.

12. Supic G, Jagodic M, Magic Z (2013) Epigenetics: a new link between nutrition and cancer. Nutr Cancer 65(6): 781-792.

13. Andreescu N, Puiu M, Niculescu M (2018) Effects of Dietary Nutrients on Epigenetic Changes in Cancer. Methods Mol Biol 1856: 121-139. 


\section{ISSN: 2574-1241}

DOI: 10.26717/BJSTR.2021.33.005454

Rashed Noor. Biomed J Sci \& Tech Res

(c) (P) This work is licensed under Creative (c) Commons Attribution 4.0 License

Submission Link: https://biomedres.us/submit-manuscript.php

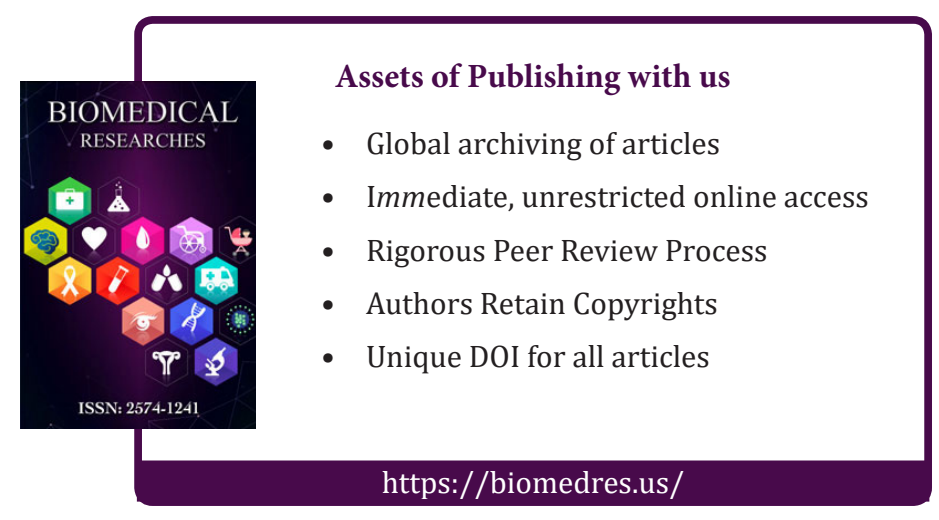

